\title{
Modelling light scattering by mineral dust using spheroids: assessment of applicability
}

\author{
S. Merikallio ${ }^{1}$, H. Lindqvist ${ }^{2}$, T. Nousiainen ${ }^{2}$, and M. Kahnert ${ }^{3}$ \\ ${ }^{1}$ Climate Change, Finnish Meteorological Institute, P.O. Box 503, 00101 Finland \\ ${ }^{2}$ Department of Physics, University of Helsinki, P.O. Box 48, 00014 Finland \\ ${ }^{3}$ Swedish Meteorological and Hydrological Institute, Folkborgsvägen 1, 60176 Norrköping, Sweden
}

Received: 17 December 2010 - Published in Atmos. Chem. Phys. Discuss.: 3 February 2011

Revised: 5 May 2011 - Accepted: 16 May 2011 - Published: 9 June 2011

\begin{abstract}
We study the applicability of spheroidal model particles for simulating the single-scattering optical properties of mineral dust aerosols. To assess the range of validity of this model, calculations are compared to laboratory observations for five different dust samples at two wavelengths. We further investigate whether the best-fit shape distributions of spheroids for different mineral dust samples have any similarities that would allow us to suggest a generic first-guess shape distribution for suspended mineral dust. We find that best-fit shape distributions vary considerably between samples and even between wavelengths, making definitive suggestions for a shape distribution difficult. The best-fit shape distribution also depends strongly on the refractive index assumed and the cost function adopted. However, a power-law shape distribution which favours those spheroids that depart most from the spherical shape is found to work well in most cases. To reproduce observed asymmetry parameters, best results are obtained with a power-law shape distribution with an exponent around three.
\end{abstract}

\section{Introduction}

The direct radiative impact of aerosols has been identified as one of the main sources of uncertainty in quantifying radiative forcing of the climate system (Forster et al., 2007). Mineral dust is one of the most widespread types of aerosol in the atmosphere with relatively high optical depth (Sokolik and Toon, 1996). In arid regions, rising concentrations

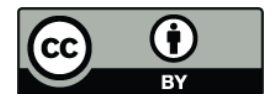

Correspondence to: S. Merikallio (sini.merikallio@fmi.fi) of mineral dust due to desertification may even constitute the dominant anthropogenic mechanism for regional radiative forcing (Myhre and Stordal, 2001). The main sources of error in quantifying the radiative impact of mineral aerosols are the refractive index (Myhre and Stordal, 2001), the nonspherical morphology (e.g., Kahnert and Nousiainen, 2006; Kahnert et al., 2007) and, to a slightly lesser extent, the size distribution (Myhre and Stordal, 2001). Accounting for aerosol morphology is also vital in remote-sensing applications (e.g., Mishchenko et al., 2007). Both radiance and, even more so, polarisation can be strongly modulated by particle nonsphericity (e.g., Mishchenko et al., 1997; Schulz et al., 1998).

In climate studies, it is still common practice to model aerosol optical properties using the homogeneous sphere approximation (HSA). Spheroidal model particles have long been investigated as a first-order improvement of the HSA (e.g., Mishchenko, 1993; Schulz et al., 1999). The idea behind this model is to introduce, in addition to the size parameter, one additional shape parameter while retaining a high degree of symmetry, thus keeping computational resource requirements manageable. Comparisons of model results and measurements indicate that spheroids are more versatile than other symmetric model particles, such as polyhedral prisms (Nousiainen et al., 2006). They have even proven superior to more advanced particle models that mimic the shape statistics of mineral dust samples (Veihelmann et al., 2006). In recent years, spheroids have been used operationally in remote sensing, such as in AERONET retrievals (e.g., Dubovik et al., 2006). Thanks to these recent successes, spheroids are likely to become established as an operational standard model for mineral dust. 
However, there are important issues that have, so far, not been adequately addressed. Validation studies have been confined to a fairly small selection of measurements. In Nousiainen et al. (2006), comparison of modelling results with measurements were limited to scattering experiments on feldspar aerosols at a wavelength of $632.8 \mathrm{~nm}$. In Dubovik et al. (2006), this validation study was repeated and supplemented by observations made for the same feldspar sample at $441.6 \mathrm{~nm}$. To increase our confidence in the spheroidal particle model, we need to perform a more comprehensive validation study, involving a larger selection of mineral dust samples with different size distributions and mineral compositions. Specifically, we need to identify the range of validity of the spheroidal particle model. For instance, recent findings suggest that spheroids in terms of single-scattering properties may not be appropriate for modelling the optical properties of highly absorbing aerosols (Rother et al., 2006) and little is known about the performance of the spheroidal model particles for mimicking scattering by dust particle ensembles with effective radii larger than about $1 \mu \mathrm{m}$. Finally, to make use of the full flexibility of spheroids, models usually employ a shape distribution of spheroids, i.e., an ensemble of spheroids with different aspect ratios. In principle, each aspect ratio in the model can have a different weight, so we could introduce as many free parameters as we have different aspect ratios in our model ensemble. Both in remote sensing and, even more so, in climate modelling applications we need to reduce the number of free parameters by introducing reasonable a priori assumptions about the shape distribution of spheroids. This raises the difficult question: Can we define a generic shape distribution that is likely to provide sufficiently accurate model results for a wide range of mineral aerosol compositions, size distributions, and wavelengths, and for different optical parameters?

Addressing these issues is pivotal for a wide range of applications within remote sensing and climate modelling. Here, we investigate these problems by performing a comprehensive validation study of the spheroidal particle model, assessing its range of validity, and studying the possibilities and limitations of generic shape distributions. In Sect. 2, we briefly review the theoretical background and in Sect. 3 we discuss the laboratory measurements employed in the comparisons. Results are presented and discussed in Sects. 4 and 5 , respectively. Work is summarized in Sect. 6 .

\section{Methodology}

We are primarily interested in modelling the elements of the phase matrix, which for randomly oriented particles has six independent elements (van de Hulst, 1957):

$$
\mathbf{P}(\theta)=\left(\begin{array}{cccc}
P_{11}(\theta) & P_{12}(\theta) & 0 & 0 \\
P_{12}(\theta) & P_{22}(\theta) & 0 & 0 \\
0 & 0 & P_{33}(\theta) & P_{34}(\theta) \\
0 & 0 & -P_{34}(\theta) & P_{44}(\theta)
\end{array}\right)
$$

Here $\theta$ denotes the scattering angle, i.e., the angle between the propagation directions of incident and scattered light. In the comparison with measurements, we consider the ratios $P_{i j} / P_{11}$ for $\{i, j\} \neq\{1,1\}$. The phase function $P_{11}$ is normalised according to

$$
\frac{1}{2} \int_{0}^{\pi} P_{11}(\theta) \sin \theta \mathrm{d} \theta=1 .
$$

The phase matrix elements are most relevant for the interpretation of remote sensing observations of radiance, polarisation, and depolarisation ratios. For climate applications, we also need to consider the asymmetry parameter $g$, which is the first Legendre moment of the phase function, i.e.,

$g=\frac{1}{2} \int_{0}^{\pi} P_{11}(\theta) \cos \theta \sin \theta \mathrm{d} \theta$.

The asymmetry parameter is a measure for the partitioning between radiation scattered in the forward and backward hemispheres, which is important for quantifying the impact of aerosols on the radiative energy budget.

The size of the particles is often described relative to the wavelength $\lambda$ of the light with a so-called size parameter $x$,

$x=\frac{2 \pi r}{\lambda}$,

where $r$ is the radius of a volume-equivalent sphere.

The geometry of the spheroidal model particles is characterised by the aspect ratio $\epsilon=a / b$, where $b$ denotes the dimension of the spheroid along the main rotational symmetry axis, and $a$ denotes the corresponding dimension perpendicular to that axis. A prolate spheroid $(\epsilon<1)$ is obtained by rotating an ellipse about its major axis, while an oblate spheroid $(\epsilon>1)$ is constructed by rotating an ellipse about its minor axis.

For parametrising the shape distribution, i.e., a distribution of spheroidal aspect ratios, it is more convenient to use a shape parameter $\xi$ (Kahnert et al., 2002a) rather than the aspect ratio $\epsilon$. The shape parameter is defined as

$\xi= \begin{cases}\epsilon-1 & : \epsilon>1 \text { (oblate) } \\ 1-1 / \epsilon & : \epsilon<1 \text { (prolate) } \\ 0 & : \epsilon=1 \text { (sphere) }\end{cases}$

If we increase $a$ for an oblate spheroid, then both $\epsilon$ and $\xi$ will increase linearly with $a$. On the other hand, if we increase $b$ for a prolate spheroid, then $\epsilon$ will decrease hyperbolically, while $\xi$ will decrease linearly with $b$. The linear $\xi$-scale lends itself more easily for parametrising the shape distribution.

Previous attempts to fit modelled or measured reference scattering matrices with a shape distribution of spheroidal model particles have consistently shown that spheroids with large values of $|\xi|$ contribute most to the best-fit ensemble (e.g., Kahnert, 2004; Nousiainen et al., 2006). For this reason, it has been suggested to parametrise the shape distribution according to a simple power law

$p(\xi)=C|\xi|^{n}, n \geq 0$ 
where the normalization factor $C$ is

$C=\int_{\xi_{\min }}^{\xi_{\max }}|\xi|^{n} d \xi$

The power law gives the largest weight on those spheroids deviating most from the spherical shape. The power-law index $n$ is an empirical parameter that has to be chosen such as to give the best agreement between modelling results and observations. AERONET shape retrievals of atmospheric dust particles reported by Dubovik et al. (2006) also resulted in a shape distribution that favored high-aspect ratio (large $|\xi|$ ) spheroids.

We make use of a database of pre-computed singlescattering properties for mineral dust particles (Dubovik et al., 2006). From the database, we can directly retrieve the scattering-matrix elements for any given aspect ratio averaged over a given size distribution within $0.012<x<625$ (Dubovik et al., 2006). In the samples there are particles whose size parameter exceeds this range. These particles are thus ignored, but their contribution to the matrix elements is estimated to be negligible. Scattering cross sections are also extracted, as they are needed for weighting when computing shape-distribution integrated quantities. The results are compared to laboratory measurements of five different samples at two wavelengths, which are further discussed in Sect. 3. The refractive indices $m$ of the samples are only known within a certain confidence range. For this reason, we perform computations for five different values of $m$ with $\operatorname{Re}(m)=1.55$ and $1.7, \operatorname{Im}(m)=0.001$ and 0.01 , and a central value of $m=1.6+0.003 i$. The feldspar sample was additionally modeled with $m=1.6+0.001 i, m=1.6+0.01 i$, $m=1.55+0.003 i$, and $m=1.7+0.003 i$. These values are based on the estimated range of $m$ provided by Volten et al. (2001) and Muñoz et al. (2001).

The model shapes include nine aspect ratios for oblate spheroids with $\epsilon=1.2,1.4, \ldots, 2.8$, and nine aspect ratios for prolate spheroids with $\epsilon=1 / 1.2,1 / 1.4, \ldots, 1 / 2.8$. This corresponds to shape parameters of $\xi=0.2,0.4 \ldots, 1.8$ (oblate), and $\xi=-0.2,-0.4, \ldots,-1.8$ (prolate). In addition, corresponding results for spheres are computed $(\epsilon=1, \xi=0)$.

The size-averaged optical properties are calculated corresponding to each of the model shapes for all five samples at both wavelengths. The volume-equivalent size is assumed. The use of area equivalence was also briefly tested, but its performance appeared to be comparable to that of the volume equivalence in reproducing the measured scattering, so further considerations using different size equivalences were deemed unnecessary to our applications. The ensembleaveraged phase matrix is obtained averaging over the 19 aspect ratios weighted by the assumed shape distribution and also by their corresponding scattering cross sections, which specify the total power scattered in all directions. Different shape distributions have been tested, with a focus on the $|\xi|^{n}$ model given in Eq. (6).

\section{Measurements}

We test our model by comparing the simulations with laboratory measurements of the scattering matrices of different dust samples. The measurements are taken from the Amsterdam Light Scattering Database (Volten et al., 2006). An example of a measured scattering matrix (with error bars) is shown in Fig. 1 along with example computations of spheroids integrated over the size distribution of the loess sample. From the samples included in the database, we chose feldspar, red clay, green clay, loess, and Saharan dust. These samples have been measured by Volten et al. (2001) except for the green clay that was measured by Muñoz et al. (2001). The size distributions of the samples have been measured using a Fritsch laser particle sizer (Konert and Vandenberghe, 1997) and are also reported in the database. Although the samples have not been collected in the atmosphere, their shapes and compositions can be considered to be representative of atmospheric dust, and their sizes cover the expected size range. Presently, no measured scattering matrices exist from samples collected from the atmosphere.

The properties of the samples are summarized in Table 1. The effective radii $\left(r_{\text {eff }}\right)$ of the samples range from $1.0 \mu \mathrm{m}$ to $8.2 \mu \mathrm{m}$ and the effective standard deviations of radius $\left(\sigma_{\mathrm{eff}}\right)$ from 1.0 to 2.0. Following Hansen and Travis (1974), these quantities are defined as

$r_{\mathrm{eff}}=\frac{\int_{r} r \cdot \pi r^{2} n(r) d r}{\int_{r} \pi r^{2} n(r) d r}$,

$\sigma_{\text {eff }}=\sqrt{\frac{\int_{r}\left(r-r_{\text {eff }}\right)^{2} \pi r^{2} n(r) d r}{r_{\text {eff }}^{2} \int_{r} \pi r^{2} n(r) d r}}$.

By replacing $r$ by $r_{\text {eff }}$ in Eq. (4), we can define the effective size parameter $x_{\text {eff. }}$.

The samples have been measured at wavelengths of $441.6 \mathrm{~nm}$ and $632.8 \mathrm{~nm}$, and cover scattering angles from $5^{\circ}$ to $173^{\circ}$. Angles from $5^{\circ}$ to $170^{\circ}$ have been measured with $5^{\circ}$ angular resolution, and angles larger than $170^{\circ}$ with $1^{\circ}$ resolution. The origins and the characteristics of the samples vary. For example, the shapes of the loess and Saharan dust are perhaps most representative of the atmospheric aerosols as they are collected from surface deposits. The feldspar sample, on the other hand, was ground from a feldspar rock and might thus be more angular than natural dust particles, but its size distribution resembles that of atmospheric dust in background conditions. The clay samples are commercial.

The measured scattering matrices, $\mathbf{F}$, are related to the phase matrix in Eq. (1) by an unknown normalisation coefficient: $\mathbf{P}=\gamma \cdot \mathbf{F}$. Both $\mathbf{F}$ and $\mathbf{P}$ are so-called Mueller matrices. The element ratios $P_{\mathrm{ij}} / P_{11}$ can thus be directly compared to the measured $F_{\mathrm{ij}} / F_{11}$, but the phase function $P_{11}$ first needs to be properly normalised according to Eq. (2). However, to compute the normalisation integral we need to have the phase function for the entire angular range from $0^{\circ}$ to $180^{\circ}$. As we 

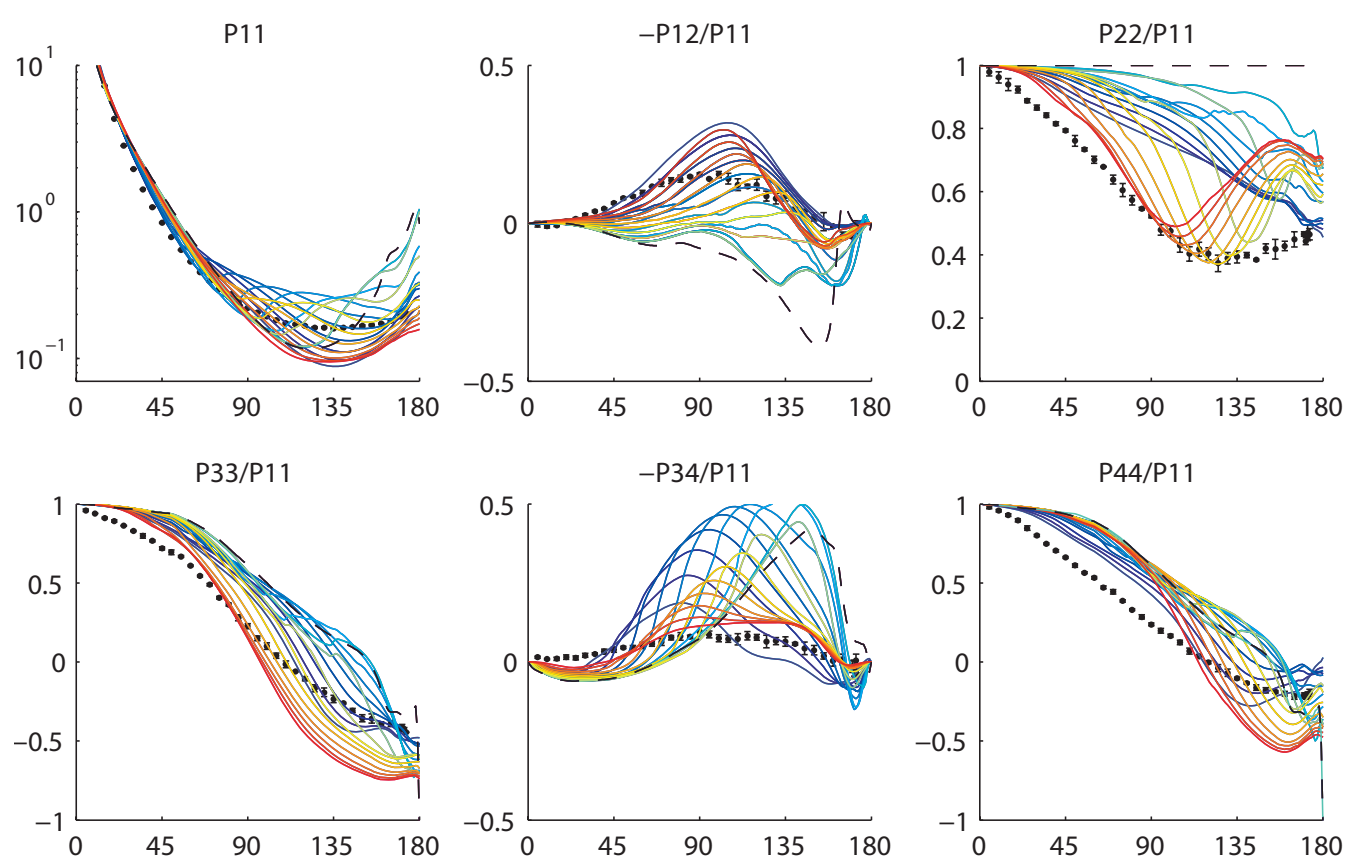

Fig. 1. Measured and simulated scattering-matrix elements for the loess sample at wavelength $\lambda=632.8 \mathrm{~nm}$. The measurements are shown with small black dots and their error bars. Different solid lines depict scattering by different spheroids with refractive index of $m=1.55+$ $0.001 i$, ranging from prolate (red) to oblate (blue) aspect. The dashed black line is the corresponding simulation for a sphere.

have no direct measurements of the forward-scattering directions, the phase function between angles from $0^{\circ}$ to $5^{\circ}$ are obtained directly from the corresponding computations. The simulated results are then matched with the observed phase function at the scattering angle $\theta=5^{\circ}$. The backscattering angles, which do not contribute much to the normalisation integral, are extrapolated simply by using the measured value at $173^{\circ}$ for all angles from $174^{\circ}$ to $180^{\circ}$. Other methods for extrapolation have been suggested, e.g., by Liu et al. (2003), Kahnert and Nousiainen (2006), and Kahnert and Nousiainen (2007).

\section{Results}

To compare simulations and measurements, we apply the measured size distribution, select a refractive index, and average the simulations over sizes and shapes as described in Sect. 2. The quality of fit is then evaluated by computing a cost function that quantifies the (dis)agreement between the simulations and measurements. As the preferred cost function, we use the area between the measured and modeled matrix elements (i.e., the well-known $l_{1}$-norm; see, e.g., Kreyszig, 1993, page 994). The area is calculated only for scattering angles at which measurements are available, and it is normalised by dividing it with the angular span of the measurements ( $\Delta \theta=168^{\circ}$ for all samples considered here), and then expressed in percentages. We name this error-quantity $\psi$ :

$\psi=\frac{100 \%}{\Delta \theta} \int_{5^{\circ}}^{173^{\circ}}\left|P_{\mathrm{obs}}-P_{\mathrm{sim}}\right| d \theta$.

Here, $P=P_{i j} / P_{11}$, except that for the phase function $P=$ $P_{11}$. The advantage here is that the errors of different scattering-matrix elements are readily comparable with each other. On the downside, the measurement uncertainty is not taken into account. If we want to give more emphasis for side scattering, we can use the $\log \left(P_{11}\right)$ instead of $P_{11}$ when calculating the $\psi$ for the phase function. Indeed, we have mainly used the logarithmic form, as it gives more even weight for all measurement angles.

We also experimented with many other cost functions, including $\chi^{2}$ errors, Eq. (A7), summed over measurement points, and the so-called $\delta_{80}$ value, which is defined such that at $80 \%$ of all observation points the discrepancy between measurements and simulations is smaller than $\delta_{80}$. In the case of $\chi^{2}$ and $\delta_{80}$ statistics, the cost function for assessing the agreement between measurements and model is calculated at the measurement points excluding $171^{\circ}, 172^{\circ}$, and $173^{\circ}$ to preserve angular equality in the analyses.

We note that we have restricted ourselves to using homogeneous, highly symmetrical model particles with smooth surfaces; real mineral particles are irregularly shaped, expected to be inhomogeneous and are likely to be composed of 
Table 1. Summary of the sample properties. The $r_{\text {eff }}$ and $\sigma_{\text {eff }}$ values have been computed from the measured size distribution; the Re $(m)$ is an estimate; $\operatorname{Im}(m)$ is estimated to be between $10^{-2}$ and $10^{-5}$ for all samples.

\begin{tabular}{|c|c|c|c|c|c|c|c|}
\hline & $r_{\mathrm{eff}}[\mu \mathrm{m}]$ & $\sigma_{\text {eff }}$ & $\operatorname{Re}(m)$ & main constituents & production & origin & colour \\
\hline feldspar & 1.0 & 1.0 & $1.5-1.6$ & K-feldspar, plagioclase, quartz & crushed & Finland & light pink \\
\hline red clay & 1.5 & 1.3 & $1.5-1.7$ & biotite, illite, quartz & commercial & France & red brown \\
\hline green clay & 1.55 & 1.2 & $1.5-1.7$ & $\begin{array}{l}\text { illite, kaolinite, montmorillonite, } \\
\text { quartz }\end{array}$ & commercial & France & green \\
\hline loess & 3.9 & 1.6 & $1.5-1.7$ & $\begin{array}{l}\text { K-feldspar, illite, quartz, calcite, } \\
\text { chlorite, albite }\end{array}$ & collected & Hungary & yellow brown \\
\hline Saharan dust & 8.2 & 2.0 & $1.5-1.7$ & $\begin{array}{l}\text { quartz, clay minerals, } \\
\text { calcium carbonate }\end{array}$ & collected & Saharan desert & yellow brown \\
\hline
\end{tabular}

birefringent and thus anisotropic mineral species (e.g., Nousiainen, 2009). Moreover, we have assumed that the particle properties are not size or shape dependent while, for real atmospheric dust particles, this is not necessarily the case. For example, Claquin et al. (1999) propose different mineralogies for clay and silt fraction particles. More recently, physical and optical properties have been measured for different size classes of airborne Saharan dust in the SAMUM campaign (Heintzenberg, 2009). Measured refractive indices were found to be varying in-between different size classes (Müller et al., 2009 and Petzold et al., 2009), which is not surprising considering that also the chemical composition was found to vary (Kandler et al., 2009 and Schladitz et al., 2009).

\subsection{Assessing the overall performance of spheroids}

We first want to establish how well the model of spheroids works for our samples. One way to do it would be to treat the shape distribution and the refractive index as free parameters, apply a fitting algorithm to find optimal values for these and then compute the cost function. However, since only positive weights are possible in the shape distribution, a nonlinear fitting algorithm must be used, and such methods are not guaranteed to locate the global minimum even when multiple initial states are used. We thus adopt a simpler method where we investigate how well the measurement points are bracketed by simulations of individual spheroidal shapes. If a measurement point lies outside the range of those matrices covered by different aspect ratios, then it is impossible to fit that measurement point with any shape distribution. This leads us to consider how well this necessary condition for successful fitting is met for different samples. The non-linear fits are only performed for selected cases and are considered in more detail in Sect. 4.2.

Investigations on how well the measured scattering-matrix elements can be covered by spheroids' of different shapes and refractive indices are thus performed. The term "coverage" refers to the percentage of measurement points that are within the range obtained by considering the spheroids size- integrated values for all aspect ratios separately. This gives an indication of how well the measurements can be modeled by using spheroids.

In Fig. 2 three scattering-matrix elements at $\lambda=632.8 \mathrm{~nm}$ have been plotted for each sample studied. Shown are both the measurement error bars and the coverages by different spheroids. The length of the error bar covered is accounted when calculating coverages, so that one single outlier point with a huge error bar might lower the coverage percentage significantly, which is exactly what happens with the feldspar

$P_{22} / P_{11}$ element in the upper right corner of Fig. 2 . The coverages averaged over all matrix elements and for the $P_{11}$ element separately are shown in Table 2 for both wavelengths. None of the measured samples are fully covered by the spheroid simulations. Feldspar stands out as the one sample that can most readily be modeled with spheroids for both wavelengths. Less than half of the measurement points for Saharan dust, on the other hand, are bracketed by the simulations, making this sample very challenging for the model of spheroids. Overall, the average coverage is better for samples with small $r_{\text {eff. }}$ Likewise, the standard deviation is smaller for samples with small $r_{\text {eff }}$, indicating that coverages are also more consistent between different phase-matrix elements for samples with small $r_{\text {eff }}$. Thus, the model of spheroids clearly seems more promising for samples with small $r_{\text {eff. On the }}$ other hand, there does not seem to be systematic differences between the wavelengths, although the effective size parameter is over $40 \%$ larger at $441.6 \mathrm{~nm}$ than at $632.8 \mathrm{~nm}$ wavelength.

In Fig. 3, the minimum $\psi$ values, Eq. (10), of all scattering-matrix elements for each sample are plotted as a function of the effective size parameter. A rising slope can be fitted to the data and its existence clearly indicates that the spheroid model works better for smaller sizes, especially in the case of the phase function. The slopes become slightly smaller if only the best-fit refractive indices for each element are considered. It is interesting to note that all the other $\psi$ values show strong dependence on size except for $P_{12} / P_{11}$ and $P_{34} / P_{11}$, which are reproduced quite well with spheroids regardless of the size range. Moreover, the minimum $\psi$ 

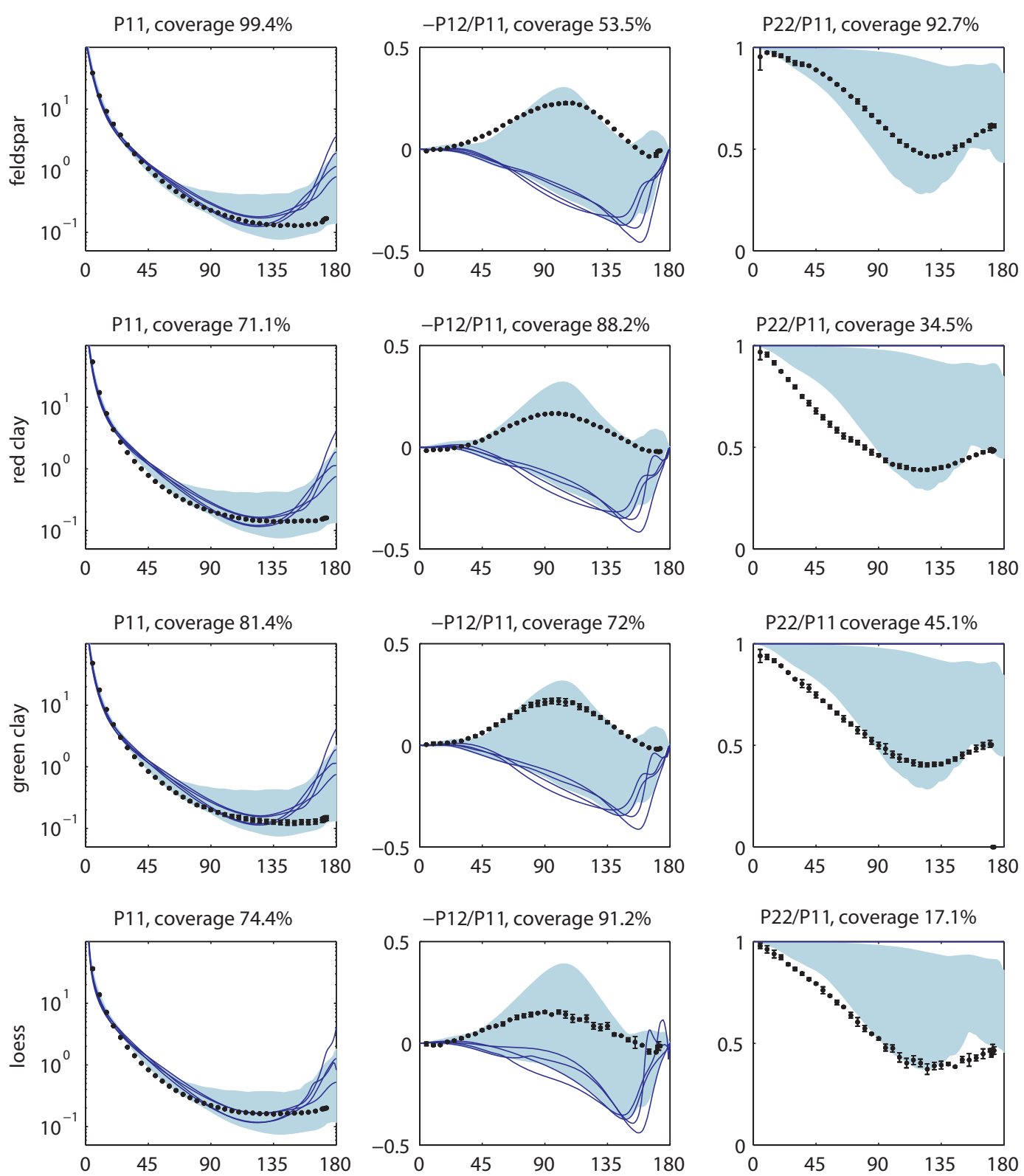

P22/P11, coverage $17.1 \%$
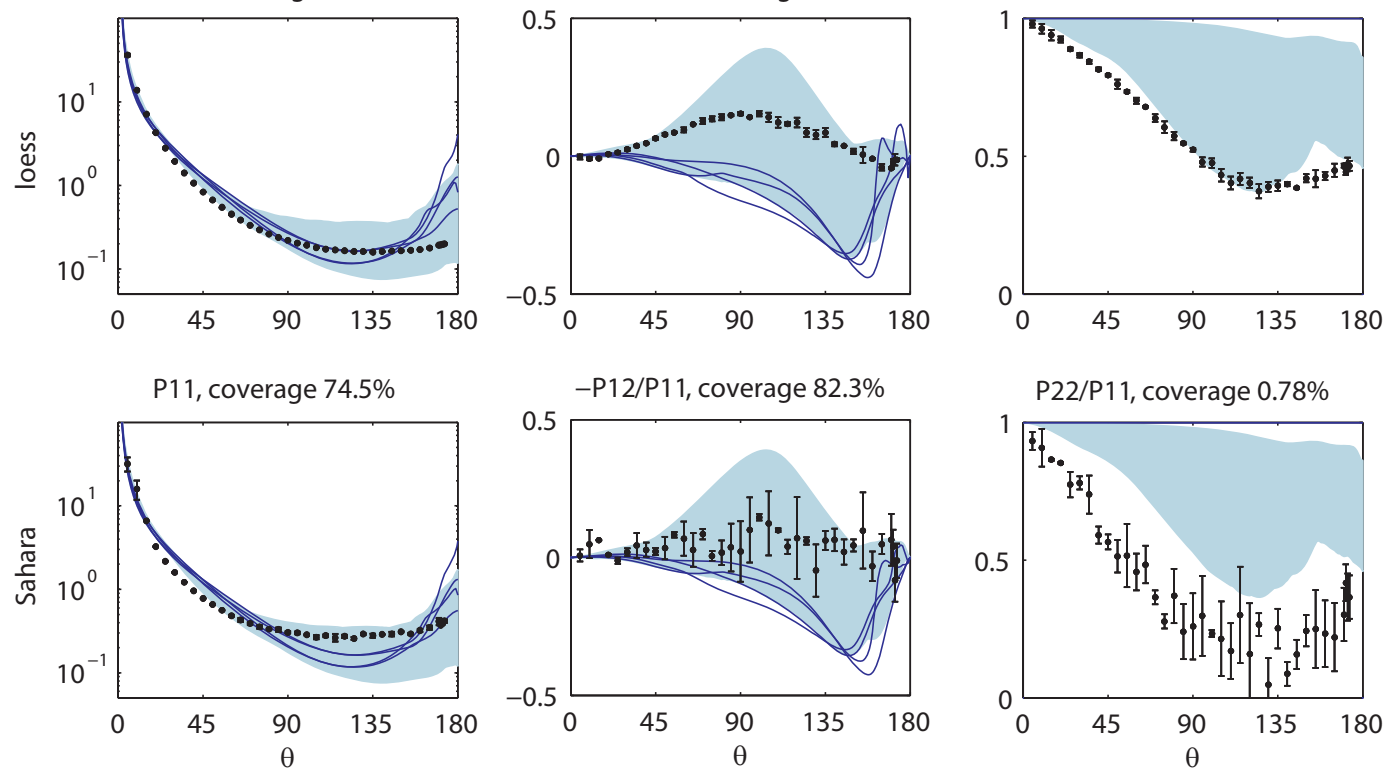

P22/P11, coverage $0.78 \%$

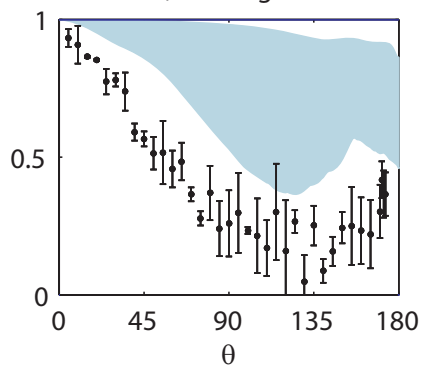

Fig. 2. Coverage of the measured scattering-matrix elements by model spheroids at $632.8 \mathrm{~nm}$ wavelength for the matrix elements $P_{11}$, $-P_{12} / P_{11}$ and $P_{22} / P_{11}$. Each row corresponds to one sample from smallest (feldspar) to the largest effective radius (Sahara). Measurements are shown with diamonds and error bars, and the shaded area indicates the coverage by different spheroids (all shapes and refractive indices), excluding spheres. The Mie spheres are shown with solid lines for each refractive index. To normalise the measured $P_{11}$ element, it has been extrapolated with the $n=3$ model shape distribution for the angles of $0^{\circ}-5^{\circ}$. 
Table 2. Percentages of spheroid coverages for different samples.

\begin{tabular}{l|ccc|ccc}
\hline$\%$ & \multicolumn{3}{|c|}{$441.6 \mathrm{~nm}$} & \multicolumn{3}{c}{$632.8 \mathrm{~nm}$} \\
\hline & $F_{11}$ & avg. & $\sigma$ & $F_{11}$ & avg. & $\sigma$ \\
feldspar & 100 & 92 & 5 & 99 & 89 & 19 \\
red clay & 72 & 62 & 24 & 71 & 58 & 24 \\
green clay & 84 & 61 & 29 & 81 & 63 & 19 \\
loess & 76 & 55 & 35 & 74 & 59 & 27 \\
Sahara & 23 & 43 & 34 & 75 & 48 & 29 \\
\hline
\end{tabular}

values of these elements do not seem to depend much on the refractive index assumed. This is probably mostly due to the extensive coverage provided to these elements by the model spheroids, allowing us to obtain good fits with different refractive indices.

\subsection{Optimal shape distributions}

Another, independent approach to assess the model of spheroids is to derive a shape distribution that provides the optimal fit to the measurements. This fit can be optimized separately for each sample, matrix element, and the refractive index. These optimized shape distributions can be found by using a nonlinear fitting algorithm based on the LevenbergMarquardt method (for detailed description, see Appendix).

Optimizing the aspect-ratio weights separately for each matrix element is a time-consuming process and was, therefore, performed for a selected set only, including all the samples and matrix elements at $\lambda=632.8 \mathrm{~nm}$ with one refractive index $(m=1.55+0.001 i)$ used for the scattering computations. In addition, fittings for the other wavelength $(\lambda=$ $441.6 \mathrm{~nm})$ and use of other refractive indices $(\operatorname{Re}(m)=1.55$ and $1.7, \operatorname{Im}(m)=0.001 i$ and $0.01 i)$ were tested for feldspar and loess samples. These represent samples with small and large $r_{\text {eff }}$; loess was chosen instead of Sahara due to its better coverage.

The fitting results (shown later in Fig. 7) reveal that in some cases, the optimal shape distribution of spheroids reproduces the measured scattering matrices quite well. As in the previous section, we once again see that the spheroids seem to work best for smaller size parameters: for feldspar, red clay, and green clay, the fits are relatively good; whereas, for loess and Saharan samples, the spheroids cannot produce scattering patterns similar to the measurements. Especially, the matrix elements $P_{22} / P_{11}$ and $P_{44} / P_{11}$ prove to be impossible to reproduce using spheroids. Fig. 3 reproduced with the optimal shape distributions (not shown) leads to a $\psi-$ $r_{\text {eff }}$ slope of 0.2 for the average of all elements as well as for that of $P_{11}$ alone.

One main goal of this study is to investigate the validity of spheroidal model particles from a broader perspective. For this reason, we are particularly interested in general trends in the optimal shape distributions. The optimal aspect-ratio

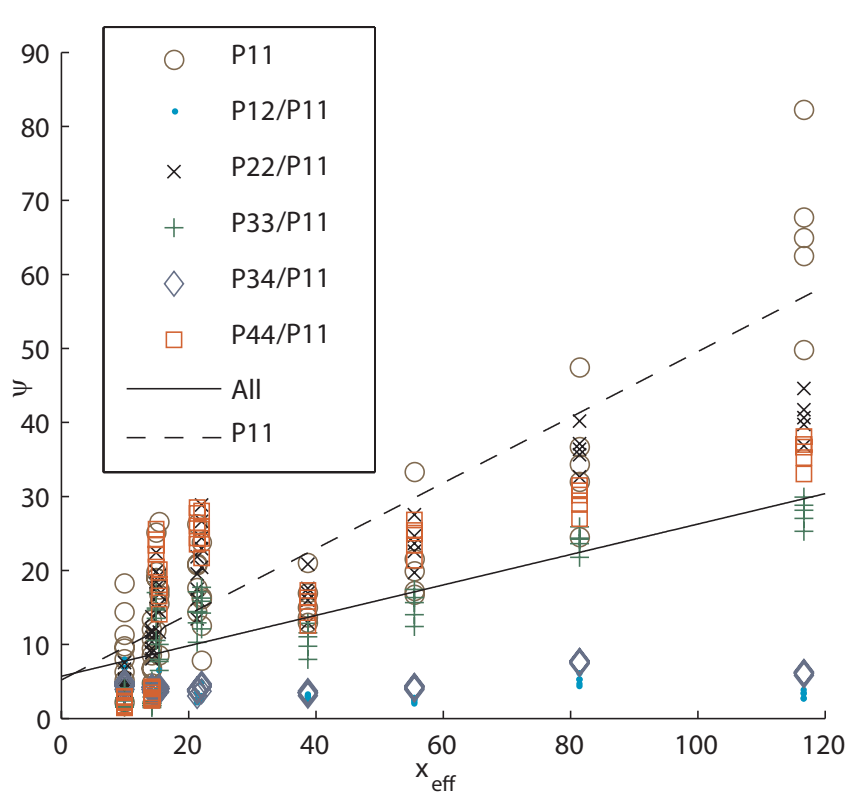

Fig. 3. Minimum $\psi$, Eq. (10), errors of the scattering-matrix elements as a function of the effective size parameter $x_{\text {eff }}$. All modeled refractive indices are included. The solid line (slope $=0.2$ ) is linear regression representing the average of all dots while the dashed line (slope $=0.4$ ) is solely for $P_{11}$.

weights for matrix elements of all samples are collected in Fig. 4. There the wavelength is taken to be $632.8 \mathrm{~nm}$ and refractive index $m=1.55+0.001 i$. An immediate conclusion on the distribution is that extreme aspect ratios are clearly most common in the best-fit shape distributions. The form of the total distribution of weights encourages us to use a powerlaw shape distribution as an a priori assumption in more detailed studies of the search for the optimal value for $n$. Hence, a power-law function $C \cdot|\xi|^{n}$ is fitted in Fig. 4 (solid black line), resulting in $n=18$. Also $n=3$ line (red) is plotted in the figure for reference. It is of interest to note that in a study by Nousiainen et al. (2006), the results favoured the extreme shapes, which in that study had $|\xi|=1.6$. Here as well, the extreme shapes are found to be strongly favoured, but as now we have included $|\xi|=1.8$, the $|\xi| \leq 1.6$ had far less weight on the results.

\section{$4.3 \xi^{n}$ parametrisation}

Nousiainen et al. (2006) suggested a simple one-parameter (n) shape distribution for modelling mineral dust based on their simulations for the feldspar sample. Here, we investigate how well such a parametrisation works in general, and to what extent the best-fit $n$ varies between the samples. To find the optimal $n$, we vary its value from 0 to 18 and identify the value that gives the smallest cost functions. At $n=18$, the very extreme shapes $(\xi=-1.8$ and 1.8$)$ include $88 \%$ of the scatterers and four most extreme shapes $(\xi=-1.8,-1.6,1.6$ 


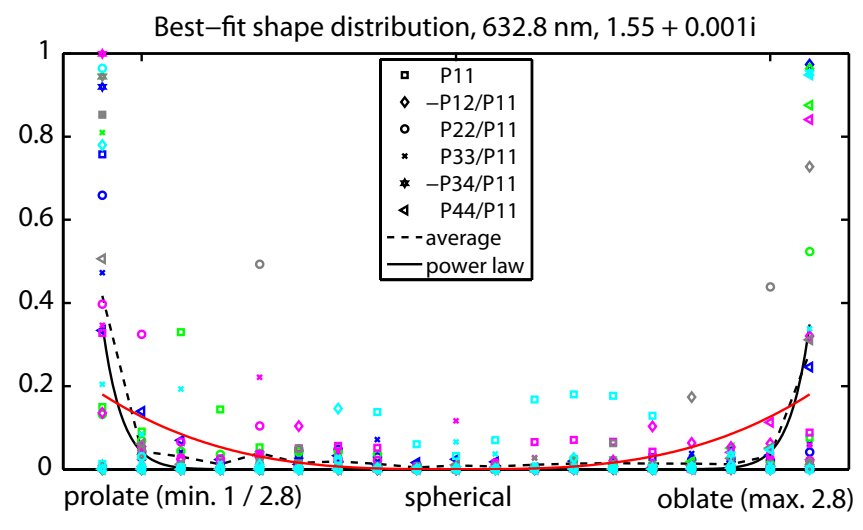

Fig. 4. Scatter plot of weights obtained for different spheroidal shapes when fitting different scattering-matrix elements of all samples considered. The colors refer to different samples: feldspar (blue), red clay (gray), green clay (green), loess (pink), and Saharan dust (black). Only the wavelength $\lambda=632.8 \mathrm{~nm}$ has been considered and $m$ has been fixed at $1.55+0.001 i$. Solid lines represent $\xi^{n}$ shape distributions with different values for $n$ : the black line shows $n=18$ that is the best value for this particular scatter plot, and the red line, corresponding to $n=3$, is shown for reference.

and 1.8 ) contain $99 \%$ of the population. The upper limit of $n=18$ was chosen to include the best-fit value of 18 obtained in the previous section. We also tested other shape distributions, which are discussed in the end of this chapter.

Table 3 summarizes the results for the optimal parametrized shape distributions under different criteria. As the cost functions, we consider eight different variations, namely:

- $\chi^{2}$ for phase function $P_{11}$;

- average $\chi^{2}$ for the independent non-zero phase matrix elements, excluding $P_{11}$;

- $\delta_{80}$ for $P_{11}$

- average $\delta_{80}$ for all independent non-zero phase matrix elements;

- the asymmetry parameter $g$;

- $\psi$ value for $\log \left(P_{11}\right)$

- average $\psi$ for all matrix elements, each with $m$ that produces the best fit;

- average $\psi$ for all matrix elements with $m$ that produces the best-fit $g$.

Obviously, best fits are obtained at different $n$ for different samples; this is natural and expected. Interestingly, with the $\chi^{2}$ criteria they are often obtained either with the least $(n=0)$ or the most $(n=18)$ extreme shape distributions considered. This is at least partly due to $\chi^{2}$ approach giving huge emphasis to few points with very small measurement errors. The $P_{11}$ element, often the most important for practical considerations, however, is uniformly best modeled with the equiprobable distribution $(n=0)$ or, when considering area differences $(\psi)$, on average with $n=0.4$. Unlike $P_{11}$, the best-fit $n$ for the asymmetry parameter $g$ is slightly larger.

There seems to be a common trend that the phase function fits best when $n$ is very small, whilst the fitting errors for polarisation components are minimized with values around $n=10$ (which is when $70 \%$ of scatterers have $|\xi|=1.8$ and $90 \%$ have $|\xi| \geq 1.6$ ) or even higher. This inconsistency indicates that the model of spheroids is not entirely accurate for real mineral dust particles.

The spheroids perform, however, much better than the homogeneous sphere approximation (HSA). This improvement in modelling accuracy is particularly clear for other samples except Sahara. The matrix elements that improve most are $P_{12} / P_{11}, P_{22} / P_{11}$ and $P_{33} / P_{11} ; \psi$ improvements are always at least $30 \%$ for the Sahara and $50 \%$ for the other samples. When the whole scattering matrix is considered, it is possible to reach $50 \%$ improvements on the average of all scattering matrix elements, excluding Saharan sample. In some special cases, individual scattering-matrix elements obtained from HSA may produce better fits, but the average $\psi$ over all matrix elements is always at least $20 \%$ better for spheroids regardless of the $n$ value or the refractive index (of those used here).

We experimented also with other kinds of shape distributions besides the $\xi^{n}$. The simplest correction, which slightly improved the results especially for small values of $n$, was to leave three or five of the most spherical shapes out altogether. Also a cosine shaped distribution was investigated, where the distribution peaked at the spherical shapes and decreased towards the more extreme axis ratios. This kind of distribution rarely matched the performance of the equiprobable distribution and was thus abandoned.

Modeled matrix elements produced by oblate particles vary from each other more than do models by prolates, which might be why shape distributions of solely oblate particles seem to produce slightly better fits to the measurements than those composed purely of prolates. A distribution that consists of both oblates and prolates usually performs best overall. It seems that both prolates and oblates are needed when good fits are sought throughout the scattering matrix for the whole angle span. Occasionally, a shape distribution tweaked into either prolate or oblate side yielded slight improvements when compared to the simple $\xi^{n}$ distribution. However, introducing an asymmetry between oblates and prolates would introduce an additional free parameter without consistent or even notable improvement to the results.

While the spheroid scheme is superior to spheres, its performance is far from perfect especially for samples with larger particles. The optimal shape distributions seem to vary from sample to sample but also, to some degree, between wavelengths. The latter implies that the optimal shape 
Table 3. The best-fit $n$ values of $\xi^{n}$ shape distributions, Eq. (6), using different criteria. The refractive index with which the best-fit value was obtained is indicated by: $a=1.55+0.001 i ; b=1.55+0.01 i ; c=1.7+0.001 i ; d=1.7+0.01 i$. and $e=1.6+0.003 i$. The last row shows the column averages when excluding the cases with $n=18$.

\begin{tabular}{l|ccccc|ccc}
\hline & $F_{11}$ & avg. & $\delta_{80} P_{11}$ & $\delta_{80}(\mathrm{avg})$. & $g$ & $\psi \log \left(P_{11}\right)$ & $\psi$ avg. best & $\psi$ all Best-g \\
\hline & & & $441.6 \mathrm{~nm}$ & & & & \\
feldspar & $0.0 \mathrm{~b}$ & $3.0 \mathrm{a}$ & $0 \mathrm{ab}$ & $0 \mathrm{ab}$ & $2.7 \mathrm{c}$ & $1.1 \mathrm{~d}$ & $2.0 \mathrm{a}$ & 6.2 \\
red clay & $0.0 \mathrm{~b}$ & $18 \mathrm{c}$ & $0 \mathrm{a}$ & $0 \mathrm{~d}$ & $1.3 \mathrm{~b}$ & $0.1 \mathrm{~b}$ & $18 \mathrm{c}$ & 8.8 \\
green clay & $0.0 \mathrm{~b}$ & $18 \mathrm{~d}$ & $0 \mathrm{c}$ & $0 \mathrm{c}$ & $1.0 \mathrm{~b}$ & $0.4 \mathrm{~b}$ & $18 \mathrm{c}$ & 6.5 \\
loess & $0.0 \mathrm{~d}$ & $18 \mathrm{c}$ & $0 \mathrm{a}$ & $0 \mathrm{~d}$ & $4.4 \mathrm{c}$ & $0.4 \mathrm{c}$ & $18 \mathrm{c}$ & 11.0 \\
Sahara & $0.0 \mathrm{c}$ & $0.0 \mathrm{c}$ & $0 \mathrm{~b}$ & $0 \mathrm{~b}$ & $0.0 \mathrm{c}$ & $0.0 \mathrm{c}$ & $1.5 \mathrm{c}$ & 9.7 \\
\hline & & & $632.8 \mathrm{~nm}$ & & & & & \\
feldspar & $6.0 \mathrm{~b}$ & $1.5 \mathrm{~b}$ & $0 \mathrm{c}$ & $0 \mathrm{c}$ & $9.4 \mathrm{a}$ & $0.7 \mathrm{~b}$ & $5.0 \mathrm{a}$ & 8.2 \\
red clay & $0.0 \mathrm{~b}$ & $18 \mathrm{~d}$ & $0 \mathrm{a}$ & $0 \mathrm{~d}$ & $3.1 \mathrm{~b}$ & $0.2 \mathrm{~b}$ & $18 \mathrm{c}$ & 9.3 \\
green clay & $0.0 \mathrm{~b}$ & $18 \mathrm{~d}$ & $0 \mathrm{c}$ & $1 \mathrm{c}$ & $3.4 \mathrm{~b}$ & $0.7 \mathrm{~b}$ & $18 \mathrm{~d}$ & 9.9 \\
loess & $0.0 \mathrm{a}$ & $0.0 \mathrm{a}$ & $0 \mathrm{~b}$ & $0 \mathrm{~b}$ & $5.7 \mathrm{e}$ & $0.1 \mathrm{a}$ & $18 \mathrm{c}$ & 7.0 \\
Sahara & $0.0 \mathrm{c}$ & $0.0 \mathrm{c}$ & $0 \mathrm{~b}$ & $0 \mathrm{~d}$ & $0.0 \mathrm{c}$ & $0.0 \mathrm{c}$ & $0.9 \mathrm{c}$ & 9.5 \\
\hline MEAN & 0.6 & 9.45 & 0 & 0.2 & 3.1 & 0.4 & 11.7 & 8.8 \\
w/o. 18 & & 0.9 & & & & 2.4 & 3.6 \\
\hline
\end{tabular}

distribution for spheroids is not unambiguously connected to the actual shapes of the particles.

\subsection{Robustness of model with respect to refractive index}

The refractive index $m$ of the samples is one of the sources of uncertainty in our analyses. Indeed, we do not even know to what degree the samples can be characterized with a single refractive index. To account for the uncertainty in $m$, simulations have been conducted with a variety of values, chosen to bracket the expected $m$ range. Still, none of the values used is likely to be exactly right for any of the samples.

One of the key questions related to this is whether the $m$ dependence of scattering is sufficiently linear over the considered interval that, when we bracket the $m$ values, we also bracket the single-scattering properties. In Nousiainen (2007), the dependence of the asymmetry parameter on the refractive index was studied for shape- and size distributions of spheroids. It was found that $g$ depends on $m$ monotonically and fairly linearly over a wide range of refractive indices. For individual, scattering-angle dependent phase matrix elements the situation is bound to be more complicated, but luckily the angular forms of the matrix elements do not seem to be overly sensitive to fairly modest variations in $m$ (e.g., Nousiainen and Vermeulen, 2003; Muñoz et al., 2007). We are thus confident that, to a large extent, we also cover the single-scattering properties in our treatment.

To estimate the sensitivity to refractive index, we take a closer look at the results for the nine different values of $m$ for the feldspar sample and the five different values of $m$ for the other samples. The summary of the results is given in Table 3. The first obvious observation is that the best-fit refractive index depends on the fitting criterion used. For example, for the feldspar sample for which the spheroid model works best, we obtain best-fit $m$ from $1.55+0.001 i$ to $1.7+0.01 i$ at $\lambda=441.6 \mathrm{~nm}$, from one extreme to the other, depending on the criterion adapted. The Saharan sample, on the other hand, favors the complementary extremes from $1.55+0.01 i$ to $1.7+0.001 i$. Behaviour is similar for $\lambda=632.8 \mathrm{~nm}$. This result strongly implies that it is very challenging to reliably invert both the optimal shape distribution and the refractive index of real dust particles from the angular dependence of the scattering-matrix elements using simple model shapes such as spheroids. The best-fit $m$ also depends on the wavelength and varies between samples, but these are expected and reasonable results.

To get more insight into the relation of the refractive index and shape distribution, we plotted cost functions bracketed over refractive indices for three samples (feldspar, red clay, and Saharan dust) in Fig. 5. The average $\psi$ error, Eq. (10), of all matrix elements and the asymmetry parameter difference are shown for the wavelength of $441.6 \mathrm{~nm}$ for three different values of $n$ (in columns). The longer wavelength behaves quite similarly and is not shown. Feldspar, whilst being clearly well mimicked with our model distributions, changes its 'best refractive index' behaviour with the changing shape distribution. On average, a combination of $m=1.55+0.001 i$ and $n=3$ works best for it, although $P_{11}$ can be best modeled with $m=1.55+0.01 i$. $\psi$ values for $P_{11}$ (not shown) and $g$ of red clay (represented in fourth row of Fig. 5) are minimized with $m=1.55+0.01 i$ for all $n$. Green clay behaves similarly to the red clay and is not shown. The behaviour for total error is more varied. Perhaps surprisingly, Saharan dust is the only particle type that shows a very consistent refractive index behaviour for all $n$, averages and $P_{11}$ (not shown) for both wavelengths. This might be partly due 

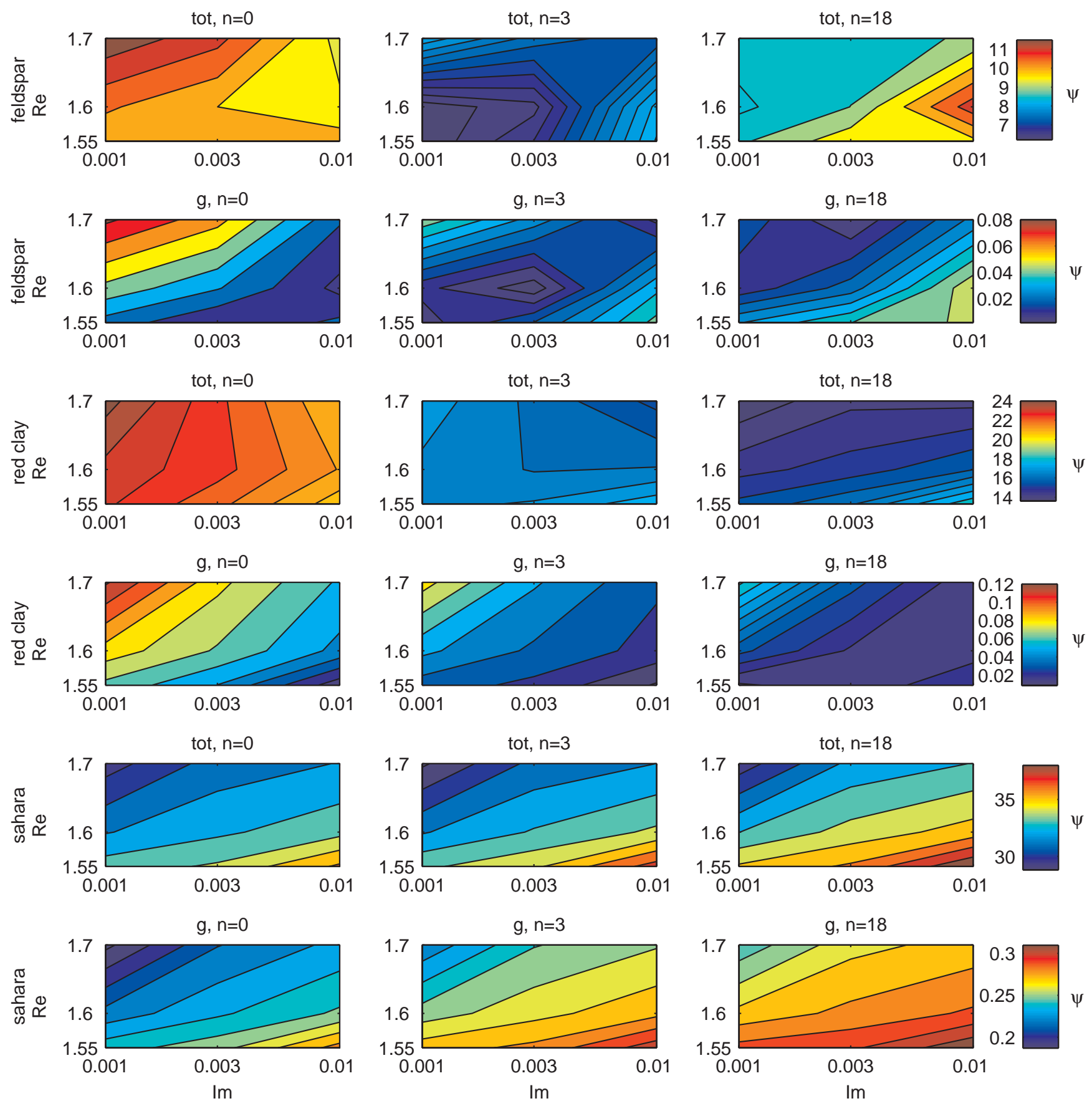

Fig. 5. Cost function dependence on the values of refractive index $m$. Columns from left to right represent different $\xi^{n}$ distributions, Eq. (6), with $n=0,3$, and 18 , respectively. Small cost function values are shown in blue whilst the worst fit values are shown in red. One should note from the colorbars that the scale varies, values increasing with increasing particle size. Three minerals are represented with $441.6 \mathrm{~nm}$, first two rows representing feldspar, then the red clay and last two rows Saharan dust. For each mineral two cost functions are shown on separate rows, namely the average $\psi$ value, Eq. (10), of all scattering-matrix elements (tot.) and that of the asymmetry parameter error $(g$ ).

to poor performance of spheroids on the Saharan sample, as large errors may mask any subtleties caused by differing refractive indices. For loess (not shown), the $m=1.7+0.001 i$ provides the best fit on the average of all the elements and also on the $P_{11}$ element for the shorter wavelength. For $632.8 \mathrm{~nm}$, the results of loess are not so conclusive as a lower real part and a higher imaginary part of the refractive index are also producing good modelling results for $g$. Overall, it 
seems that out of our options, reasonably good choices for refractive indices would be $m=1.55+0.001 i$ for feldspar, $m=1.55+0.01 i$ for both clays, and $m=1.7+0.001 i$ for both loess and Sahara.

Finally, we tested whether the matrices could be fitted better using a linear combination of different refractive indices rather than a single, fixed value. Thus, we assumed that the samples could be composed of multiple dust modes with their unique refractive indices; however, for simplicity, each mode was assumed to have the same shape and size distribution. More detailed considerations are outside the scope of this study. For comparison, we also calculated the refractive indices that produce the worst fits.

Curiously, none of the best or worst fits include the middlemost of our refractive index values, $m=1.6+0.003 i$. The reason for this is that our modeled scattering-matrix elements in most of the cases fall on one or the other side of the measurements, so that the most extreme modelling results will always be favoured with $100 \%$ concentration. This might indicate a problem with the overall suitability of the spheroid approach on real mineral dust. For example, if spheroids are incapable of producing sufficiently strong depolarisation or tend to under- or overestimate linear polarisation, it would be natural for the distribution to favour the $m$ value that produces scattering matrices with the smallest error. The $m$ value thus retrieved might have little to do with the actual $m$ value of the sample.

Alternatively, it could also be that the behaviour is connected to possible size distribution errors; it is well known that accurate measurements of size distribution are notoriously difficult (Reid et al., 2003). Then again, the size dependence of the phase matrix elements for shape-averaged spheroids is not strong. This can be seen very clearly from Fig. 9 of Nousiainen (2009), where the simulated phase matrices for the same samples as considered here are shown. In each case, the refractive index and the shape distribution has been the same, so the only differences between the samples are their different size distributions. As can be seen, the phase matrices are very similar. Therefore, errors in the size distributions are not expected to be critical for the results obtained here.

\section{Generic shape distribution}

Spheroidal model particles are a promising alternative to homogeneous spheres for both climate forcing and remote sensing applications. As a model geometry, spheroids are significantly more flexible and provide, in most cases, more accurate results for the optical properties of mineral aerosols than homogeneous spheres. In the preceeding sections we have investigated the versatility, but also the limitations, of the spheroidal particle model by performing a comprehensive validations study. In this section we determine whether we can give specific recommendations for a generic shape distribution of spheroidal particles that would provide sufficiently accurate results for a wide range of mineral dust samples, spectral bands, and for different optical parameters. One challenge in using spheroidal model particles in operational applications, e.g. in a climate model, is that the shape distribution introduces many free parameters (as many as we have discrete aspect ratios in our ensemble of model particles). Also, possible shape distribution differences between available measured samples and real atmospheric dust lead us to seek for a generic shape distribution that would work for a large range of dust particles thus also including those in the atmosphere. By specifying an a priori shape distribution, and by averaging the optical properties over this shape distribution, we reduce the free parameters to the particle size and refractive index, just like in the homogeneous sphere model. So replacing lookup tables based on spheres by those based on spheroids would be quite straightforward.

In satellite remote sensing, it may be possible to optimize the shape distribution to get best agreement with the measurements. However, it may be questioned how meaningful it is to perform fitting of optical observations with such a large set of free parameters. In climate models, on the other hand, such a fitting procedure is not even possible in principle. In the future, there might be source-dependent shape information available for climate modelling, but the authors are not aware of any such data being available currently. Further, as shown here, the connection between the real shapes and the best-fit shape distribution of spheroids may not be clear. Therefore, a generic shape distribution might be very usable for climate modelling purposes. For such a purpose, it is best to use a criterion that optimizes the asymmetry parameter, as $g$ is a key parameter in computing radiative fluxes (e.g., Kahnert et al., 2005).

By taking the average of the shape distribution $n$ values that minimize the error of the asymmetry parameter for the best performing refractive index for each particle and wavelength, we get $n=2.9$. If only the clays and feldspar are taken into account, the distribution becomes slightly steeper: $n=3.2$. The standard deviations between different samples, however, are notably large, namely 3 in both cases, meaning that quite likely the generic shape distribution is only able to portray different populations on average. Interestingly, the feldspar scattering matrix is, on average, best minimized with $n=3.0$ for $441.6 \mathrm{~nm}$ and $n=2.5$ for $632.8 \mathrm{~nm}$.

In Fig. 6, $\psi$ values obtained from the comparisons of simulations and measurements are illustrated. For each of the samples, we have used only one well performing refractive index, same for both wavelengths. For feldspar we used $m=$ $1.55+0.001 i$, for red clay and green clay $m=1.55+0.01 i$, and for loess and Sahara $m=1.7+0.001 i$. Wider bars correspond to the wavelength of $632.8 \mathrm{~nm}$, whilst the thinner black bars on top of them represent $441.6 \mathrm{~nm}$. Each row corresponds to one sample from smallest (feldspar) to the largest effective radius (Sahara). Three different representations of the $\xi^{n}$ distribution are shown for each scattering-matrix 

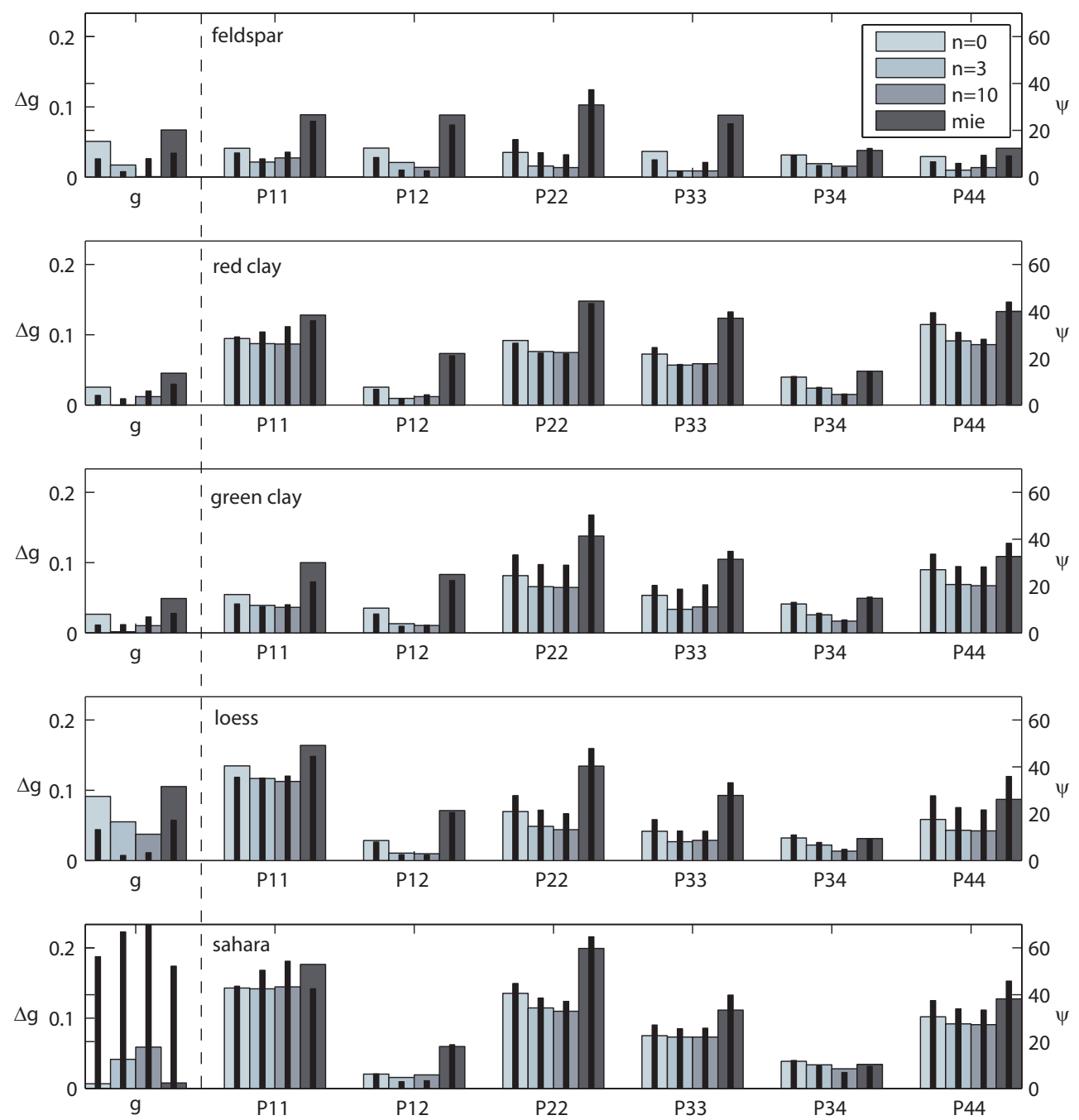

Fig. 6. $\psi$ values, Eq. (10), for model scattering-matrix elements and asymmetry parameter error compared to the measurements. Wider bars correspond to the wavelength of $632.8 \mathrm{~nm}$, whilst the thinner bars represent $441.6 \mathrm{~nm}$. Each row corresponds to one sample from the smallest (feldspar) to the largest effective radii (Sahara). On each row there are seven bar groups. The left-most group and the left y-axis shows the asymmetry parameter error, whilst the other groups and the right y-axis are for different scattering-matrix element $\psi$ values. In each group three different representations of the $\xi^{n}$ distribution, Eq. (6), namely $n=0,3$ and 10, are shown. The darkest rightmost bar represents the $\psi$ value obtained when using Mie spheres.

element, from lightest bars $(n=0)$ darkening towards $n=3$ and $n=10$ as indicated in the legend. The darkest rightmost bar represents $\psi$ obtained when using HSA. Logarithmic area difference was also investigated in the case of $P_{11}$ element, but it produced consistent results with the linear approach, so we left it out of the figure. It can be seen that, in almost all cases throughout the matrix elements, the $\xi^{n}$ models work better than the Mie solution (HSA) regardless of the $n$ used. Only exceptions are seen in the $P_{34} / P_{11}$ element of loess and Sahara sample, for which the Mie spheres perform slightly better than the equiprobable $(n=0)$ and $n=3$ distributions, and in the Saharan samples $P_{11}$ element at $441.6 \mathrm{~nm}$, which is the only case when the Mie solution is the best op- tion. This confirms that using any reasonable distribution of spheroids tends to produce better results than the Mie scheme. When the asymmetry parameter is the criterion, a reasonable first assumption for a spheroid shape distribution is to use the power law function with $n=3$. For the polarisation elements it might prove profitable to favour heavily the most extreme shapes $(n=18$, which is the maximum used in our analyses).

When using a generic shape distribution ( $n=3$ distribution) to describe the optical properties of any of our samples, the improvements compared to using HSA are generally huge. Only for the Saharan sample do the spheroids fail to decrease the error on asymmetry parameter from that 

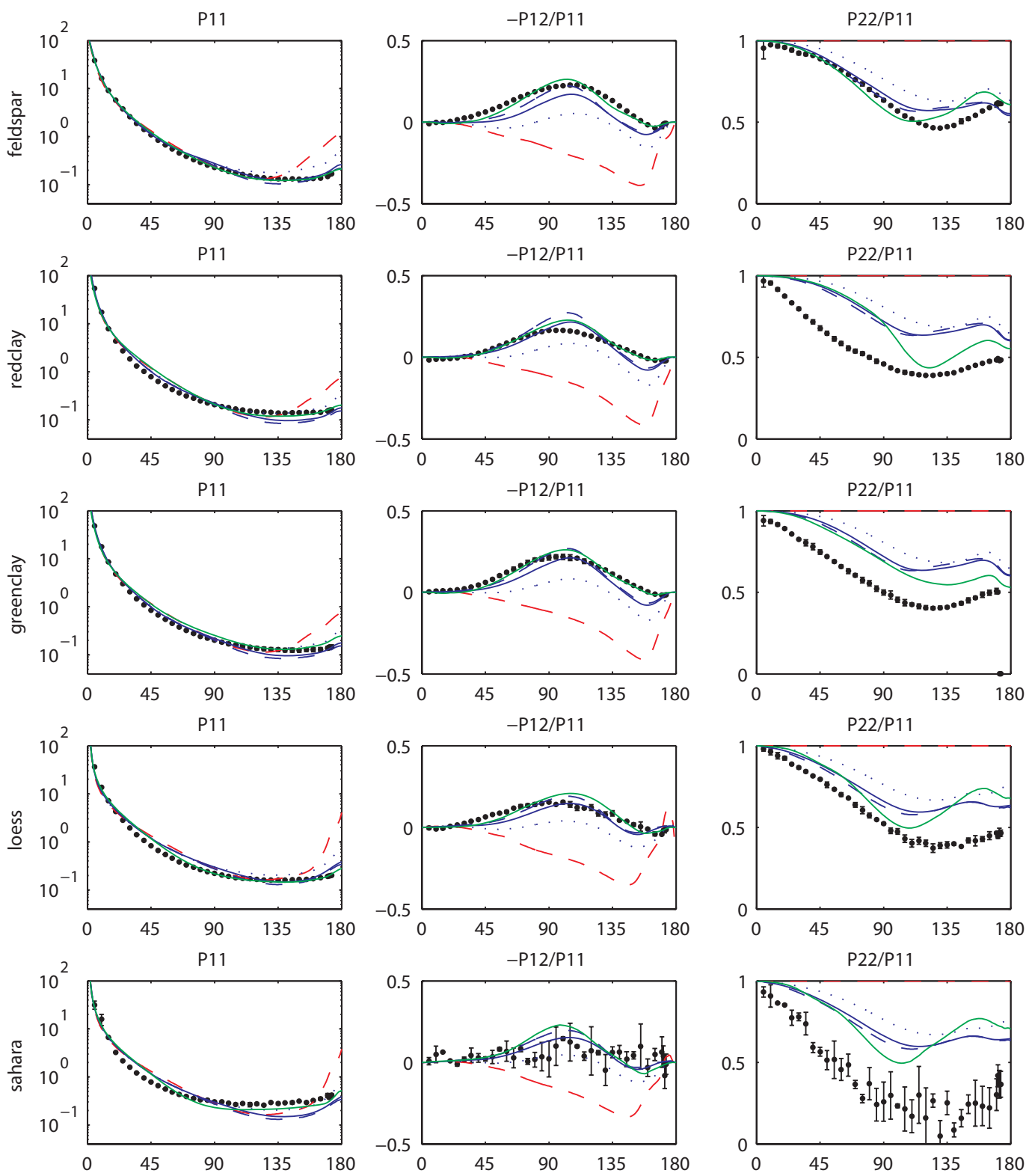

Fig. 7. Measurements with error bars (black), spheroid models (blue and green), and HSA (red) shown for the three key scattering-matrix elements of every sample. Three $\xi^{n}$ model runs, $n=0$ (dotted blue line), 3 (solid blue line), and 10 (dashed blue line) are shown as well as the optimal shape distribution (solid green line). Here we have used $\lambda=632.8 \mathrm{~nm}$.

produced by Mie particles. For the other particles, spheroids decrease the Mie error by $60-100 \%$ (60\% for green clay at $441.6 \mathrm{~nm}, 70 \%$ for red clay at $441.6 \mathrm{~nm}$ and more than $85 \%$ for both wavelengths of loess. Feldspar for both wavelengths and clays at $632.8 \mathrm{~nm}$ all have $100 \%$ improvement, meaning that the model successfully reproduces the measured asymmetry parameter).

Performance of spheroids is illustrated in Fig. 7, where three key scattering-matrix elements are shown for all samples. Measurements, spheroids with $n=0,3$, and 10, the homogeneous sphere approximation, and the optimal shape distribution results (Sect. 4.2) are plotted. It should be noted that the optimal distribution is acquired independently for all matrix elements, making the comparisons to the $\xi^{n}$ model somewhat unfair. For all fitted shape distributions in the figure (green lines), the refractive index $m=1.55+0.001 i$ has been used. For the $\xi^{n}$ models, on the other hand, we have always used $m$ that has been deemed best for the sample overall (see Sect. 4.4). This has led us to use $m=$ $1.55+0.001 i$ for feldspar, $m=1.55+0.01 i$ for both clays and $m=1.7+0.001 i$ for loess and Saharan samples. When comparing this figure with the values in Table 3 , it is noted 
that the observed behaviour differs in some respects because of the used refractive indices. For example, while in Table 3 it can be seen that $m=1.55+0.001 i$ provides the smallest error for loess $P_{11}$ with $n=0$, the behaviour seen in Fig. 7 demonstrates how the choice of a higher real refractive index, here $m=1.7+0.001 i$, also leads to a preference of different, in this case of a larger $n$. Overall, it can be seen that the optimally tuned elementwise shape distributions do not lead into universally better results although locally the improvements might be notable.

\section{Summary and conclusions}

We started our investigation by identifying three open problems regarding the use of spheroidal model particles in remote sensing and climate modelling. We wanted to (i) perform a more comprehensive validation study to test the applicability of spheroids to modelling mineral dust optical properties; (ii) stake out the range of validity of the spheroidal particle model; and (iii) investigate if we can find a generic shape distribution of spheroids that is applicable to a broad range of mineral dust samples. To address these questions we have used shape distributions of spheroids to reproduce the scattering matrix elements measured in a laboratory for five different mineral dust samples at two wavelengths. We have made use of a database of pre-computed single-scattering properties for spheroids by Dubovik et al. (2006). The measured scattering matrix elements, as well as the size distributions and the estimated ranges for the complex refractive indices of the samples of interest, have been obtained from the Amsterdam Light Scattering Database (Volten et al., 2006). The volume-equivalent size has been assumed.

Our results indicate that earlier validation studies that were limited to feldspar aerosols may have overestimated the versatility of spheroids for modelling mineral aerosol optical properties. This is especially true for mineral dust samples with larger effective size parameters. Measurements of the smallest particles can most readily be reproduced whilst the scattering matrices of largest particles are more difficult, often impossible, for spheroids to mimic. There are also differences in how the model fares on different scattering matrix elements. For example, a generally poor reproduction of $P_{22}$ element with spheroids indicates strong limitations in predicting depolarisation properties of real dust particles.

We have also analysed the best-fit shape distributions for the samples at both wavelengths. We have used a non-linear fitting algorithm to find optimal shape distributions. The merit of this approach is to (i) obtain an upper bound for how faithfully the spheroidal particle model can fit the measurements; and (ii) try to find a general pattern in the best-fit shape distributions, which can help in the development of a generic shape distribution that could be used for atmospheric dust in cases when optimisation is not possible and no additional information about dust particles is available. The re- sults indicate that shape distributions that put more weight on the most extreme aspect ratios often, but not always, provide the best fits of the measurements.

Based on this observation, we have investigated the performance of a simple one-parameter power-law shape distribution, Eq. (6). Other types of shape distributions, some with more free parameters, were also considered, but they did not result in any significant or consistent improvements. Accordingly, the best-fit power-law shape distributions for different samples at different wavelengths have been compared. The impact of using a different size equivalence would most likely not have extended beyond minor details in the results. In particular, it is noted that different size equivalences weight different aspect ratios differently, which can be partially compensated by the shape distribution weights, thus the retrieved values of $n$ might be somewhat affected.

Although relatively good results can be acquired by various shape distributions, it turns out that it is not possible to suggest a single shape distribution that would be the best choice in all cases. Not only does the best-fit distribution vary between the samples, but it also varies between the wavelengths, the metrics used for specifying the goodness of fit, the quantities fitted, and the refractive index assumed. While it is rather reasonable that the best-fit distributions would be different for different samples that can consist of differently shaped dust particles, it is disconcerting that it also depends on the wavelength. This implies that the best-fit shape distributions do not necessarily correlate with the actual dust particle shapes. Indeed, these findings suggest that, when inverting dust physical properties from the single-scattering properties, the use of simplified model shapes, such as spheroids, may lead to erroneous results even when the agreement is good - the smallness of the residuals in the fitting may not guarantee the accuracy or correctness of the results.

Despite all shortcomings of the spheroidal particle model that this study revealed, our results confirm that spheroids are superior to the homogeneous sphere approximation (HSA) in almost all cases. Also, for climate modelling purposes, in which we mainly try to overcome the inaccuracy of the HSA, a shape distribution with $n=3$ seems to be a reasonable choice. This distribution tends to produce significantly more accurate asymmetry parameter values than the HSA approach. We thus suggest a $n=3$ distribution to be used in climate models. When one wishes to optimise the phase function, an equiprobable $(n=0)$ or a very low value of $n(n<1)$ seems to perform better. When, on the other hand, one aims at the best all-around reproduction of the scattering-matrix, the optimal value of $n$ often raises significantly; in half of our cases right up to our upper limit of $n=18$. Also, the best-fit shape distributions obtained using the non-linear fitting algorithm resemble high- $n$ shape distributions. 
Recently, a database of single-scattering properties for triaxial ellipsoidal mineral dust aerosols has emerged (Meng et al., 2010). Using tri-axial ellipsoids could possibly be the next logical step towards better operational aerosol modelling, although having all three principal axes differing from each other increases the complexity of the model. However, although most likely further enhancing the fits, these new model shapes do not necessarily bring any more reliability into retrievals, as their shapes are almost as distant from the real dust particle shapes as spheroids are. It is thus suggested that inversion algorithm developers used other criteria in addition to small residuals to validate the retrievals. There are also other promising shapes currently studied elsewhere, e.g., Poisson-Voronoi tessellation (Ishimoto et al., 2010) and nonsymmetric hexahedra (Bi et al., 2010), but single-scattering properties appear not to be currently available for sufficient size- and wavelength range to consider climate model applications, for example.

\section{Appendix A}

\section{Fitting of the Mueller matrix}

Suppose we have a reference sample of particles with known optical properties. The Mueller matrix elements $P_{i, j}\left(\theta_{k}\right)$ have been measured at discrete angles $\theta_{1}, \ldots, \theta_{K}$. The corresponding standard deviations of these measurements are denoted by $\sigma_{i, j, k}$. The scattering cross section $C_{\text {sca }}$ can be obtained by determining the size-distribution and refractive index of the sample, and by performing Lorenz-Mie computations.

Suppose further that we have a set of model particles, such as spheroids of different shape parameters $\xi_{1}, \ldots, \xi_{L}$ with corresponding Mueller matrix elements $P_{i, j}^{\operatorname{sim}}\left(\theta ; \xi_{l}\right)$, and with scattering cross sections $C_{\mathrm{sca}}^{\operatorname{sim}}\left(\xi_{l}\right), l=1, \ldots, L$. Given a shape-mixture with distribution function $p(\xi)$, the averaged optical properties of an ensemble of model particles are given by

$$
\begin{aligned}
\left\langle P_{i, j}^{\operatorname{sim}}(\theta)\right\rangle & =\frac{1}{\left\langle C_{\mathrm{sca}}^{\operatorname{sim}}\right\rangle} \int_{0}^{\infty} p(\xi) C_{\text {sca }}(\xi) P_{i, j}^{\operatorname{sim}}(\theta ; \xi) \mathrm{d} \xi \\
\left\langle C_{\mathrm{sca}}^{\operatorname{sim}}\right\rangle & =\int_{0}^{\infty} p(\xi) C_{\mathrm{sca}}(\xi) \mathrm{d} \xi
\end{aligned}
$$

In discrete form this becomes

$$
\begin{aligned}
\left\langle P_{i, j}^{\operatorname{sim}}(\theta)\right\rangle & =\frac{1}{\left\langle C_{\mathrm{sca}}^{\operatorname{sim}}\right\rangle} \sum_{l=1}^{L} p_{l} C_{\mathrm{sca}}\left(\xi_{l}\right) P_{i, j}^{\operatorname{sim}}\left(\theta ; \xi_{l}\right) w_{l}, \\
\left\langle C_{\mathrm{sca}}^{\operatorname{sim}}\right\rangle & =\sum_{l=1}^{L} p_{l} C_{\mathrm{sca}}\left(\xi_{l}\right) w_{l},
\end{aligned}
$$

where the coefficients $w_{l}$ denote the integration weights of the numerical integration method employed.
The objective is to optimise the shape distribution weights $p_{l}$ such that the differential scattering behaviour of the ensemble of model particles mimics that of the reference sample as closely as possible. More specifically, we want to fit the quantity

$$
B_{i, j}^{\prime}\left(\theta ; p_{1}, \ldots, p_{L}\right)=\sum_{l=1}^{L} p_{l} C_{\mathrm{sca}}\left(\xi_{l}\right) P_{i, j}^{\operatorname{sim}}\left(\theta ; \xi_{l}\right) w_{l}
$$

to the quantity

$$
A_{i, j}(\theta)=C_{\mathrm{sca}} P_{i, j}(\theta)
$$

by optimising the weights $p_{l}, l=1, \ldots, L$. Note that this approach is an extension of earlier work Kahnert et al. (2002b); Kahnert (2004); Nousiainen et al. (2006). The main difference is that we previously fitted the Muller matrix elements themselves, whereas in the present work we fit the Mueller matrix elements scaled by the scattering cross section. The former approach is equivalent to the latter only if the model particles and the reference system all have the same scattering cross section. Ideally, the measure employed for defining size-equivalence of nonspherical particles would ensure that nonspherical particles of equivalent sizes have the same scattering cross section. In practice, this is not always the case. For this reason, the approach employed in this study is slightly more accurate than that employed in our earlier work.

The linear least-squares method solves the fitting problem by minimising the quantity

$\chi^{2}=\sum_{k=1}^{K}\left(\frac{A_{i, j}\left(\theta_{k}\right)-B_{i, j}^{\prime}\left(\theta_{k} ; p_{1}, \ldots, p_{L}\right)}{\sigma_{i, j, k}}\right)^{2}$.

A problem is that the weights $p_{1}, \ldots, p_{L}$ should have the properties of probabilities, i.e., they have to satisfy the constraints

$$
\begin{gathered}
\sum_{l=1}^{L} p_{l} w_{l}=1 \\
0 \leq p_{l} \leq 1 .
\end{gathered}
$$

These constraints can be enforced by replacing the expression in Eq. (A5) by

$$
B_{i, j}\left(\theta ; h_{1} 2, \ldots, h_{L} 2\right)=\frac{\sum_{l=1}^{L} h_{l} 2 C_{\mathrm{sca}}\left(\xi_{l}\right) P_{i, j}^{\operatorname{sim}}\left(\theta ; \xi_{l}\right) w_{l}}{\sum_{m=1}^{L} h_{m}^{2} w_{m}} .
$$

The weights $h_{1}, \ldots, h_{L}$ are determined by minimising the quantity

$$
\chi^{2}=\sum_{k=1}^{K}\left(\frac{A_{i, j}\left(\theta_{k}\right)-B_{i, j}\left(\theta_{k} ; h_{1} 2, \ldots, h_{L} 2\right)}{\sigma_{i, j, k}}\right)^{2} .
$$

Finally, one sets

$$
p_{l}=\frac{h_{l} 2}{\sum_{m=1}^{L} h_{m}^{2} w_{m}} .
$$


Equation (A12) ensures that the constraints given in Eqs. (A8) and (A9) are satisfied. Substitution of Eq. (A12) into Eq. (A10) yields an expression on the right hand side that is formally identical to that of Eq. (A5). However, in Eqs. (A10) and (A12) the coefficients $p_{l}$ are forced to be non-negative and normalised to unity.

Finally the optimised weights $p_{l}$ are substituted into Eqs. (A3) and (A4) to obtain the best-fit Mueller matrix and scattering cross section of the ensemble of model particles. Note that Eq. (A7) defines a linear least-squares problem. By contrast, Eq. (A11) needs to be solved with non-linear least-squares minimisation techniques. We employed a standard approach for such problems known as the LevenbergMarquardt method (Press et al., 1992). Note further that the least-squares technique requires that the number of known quantities should be at least twice as large as the number of unknowns. Thus, the number of scattering angles, at which observations are available, should be at least twice as large as the number of model particles in the ensemble, i.e., $K \geq 2 L$. In our case, $K=37$, and $L=17$, so the condition is satisfied.

Acknowledgements. We thank O. Munoz and H. Volten for their measurements and O. Dubovik for the use of his database, both of which were vital to this study. We acknowledge the funding by the Academy of Finland (contracts 125117, 125180 and 121482). M. Kahnert acknowledges funding from the Swedish Research Council under contract 80438701.

Edited by: Y. Balkanski

\section{References}

Bi, L., Yang, P., Kattawar, G. W., and Kahn, R.: Modeling optical properties of mineral aerosol particles by using nonsymmetric hexahedra, Appl. Opt., 49, 334-342, doi:10.1364/AO.49.000334, http://ao.osa.org/abstract.cfm? URI=ao-49-3-334, 2010.

Claquin, T., Schultz, M., and Balkanski, Y.: Modeling the mineralogy of atmospheric dust sources, J. Geophys. Res., 104, 2224322256, 1999.

Dubovik, O., Sinyak, A., Lapyonok, T., Holben, B. N., Mishchenko, M., Yang, P., Eck, T. F., Volten, H., Muñoz, O., Veihelmann, B., van der Zande, W. J., Leon, J.-F., Sorokin, M., and Slutsker, I.: Application of spheroid models to account for aerosol particle nonsphericity in remote sensing of desert dust, J. Geophys. Res., 111, D11208, doi:10.1029/2005JD006619, 2006.

Forster, P., Ramaswamy, V., Artaxo, P., adn R. Betts, T. B., Fahey, D., Haywood, J., Lean, J., Lowe, D. C., Myhre, G., Nganga, J., Prinn, R., Raga, G., Schulz, M., and Dorland, R. V.: Changes in atmospheric constituents and in radiative forcing, in: Climate Change 2007: The Physical Science Basis, edited by: Solomon, S., Qin, D., Manning, M., Chen, Z., Marquis, M., Averyt, K., Tignor, M., and Miller, H., Contribution of Working Group I to the Fourth Assessment Report of the Intergovernmetal Panel on Climate Change, Cambridge University Press, Cambridge, 2007.
Hansen, J. E. and Travis, L. D.: Light Scattering in Planetary Atmospheres, Space Sci. Rev., 16, 527-610, 1974.

Heintzenberg, J.: The SAMUM-1 experiment over Southern Morocco: overview and introduction, Tellus B, 61, 2-11, doi:10.1111/j.1600-0889.2008.00403.x, 2009.

Ishimoto, H., Zaizen, Y., Uchiyama, A., Masuda, K., and Mano, Y.: Shape modeling of mineral dust particles for light-scattering calculations using the spatial Poisson Voronoi tessellation, J. Aerosol Sci., 41, 501-512, 2010.

Kahnert, F. M.: Reproducing the optical properties of fine desert dust aerosols using ensembles of simple model particles, J. Quant. Spectrosc. Radiat. Transfer, 85, 231-249, 2004.

Kahnert, F. M., Stamnes, J. J., and Stamnes, K.: Can simple particle shapes be used to model scalar optical properties of an ensemble of wavelength-sized particles with complex shapes?, J. Opt. Soc. Am. A, 19, 521-531, 2002a.

Kahnert, F. M., Stamnes, J. J., and Stamnes, K.: Using simple particle shapes to model the Stokes scattering matrix of ensembles of wavelength-sized particles with complex shapes: Possibilities and limitations, J. Quant. Spectrosc. Radiat. Transfer, 74, 167182, $2002 b$.

Kahnert, M. and Nousiainen, T.: Uncertainties in measured and modelled asymmetry parameters of mineral dust aerosols, J. Quant. Spectrosc. Radiat. Transfer, 100, 173-178, doi:10.1016/j.jqsrt.2005.11.035, 2006.

Kahnert, M. and Nousiainen, T.: Variational data analysis method for combining laboratory-measured light scattering phase functions and forward-scattering computations, J. Quant. Spectrosc. Radiat. Transfer, 103, 27-42, doi:10.1016/j.jqsrt.2006.07.011, 2007.

Kahnert, M., Nousiainen, T., and Veihelmann, B.: Spherical and spheroidal model particles as an error source in aerosol climate forcing and radiance computations: A case study for feldspar aerosols, J. Geophys. Res., 110, D18S13, doi:10.1029/ 2004JD005558, 2005.

Kahnert, M., Nousiainen, T., and Räisänen, P.: Mie simulations as an error source in mineral aerosol radiative forcing calculations, Q. J. R. Met. Soc., 133, 299-307, 2007.

Kandler, K., Schütz, L., Deutscher, C., Ebert, M., Hofmann, H., Jäckel, S., Jaenicke, R., Knippertz, P., Lieke, K., Massling, A., Petzold, A., Schladitz, A., Weinzierl, B., Wiedersohler, A., Zorn, S., and Weinbruch, S.: Size distribution, mass concentration, chemical and mineralogical composition and derived optical parameters of the boundary layer aerosol at Tinfou, Morocco, during SAMUM 2006, Tellus B, 61, 32-50, doi:10.1111/j.16000889.2008.00385.x, 2009.

Konert, M. and Vandenberghe, J.: Comparison of laser grain size analysis with pipette and sieve analysis: a solution for the underestimation of the clay fraction, Sedimentology, 44, 532-535, 1997.

Kreyszig, E.: Advanced Engineering Mathematics, John Wiley \& Sons, 7 Edn., 1271 pp., 1993.

Liu, L., Mishchenko, M. I., Hovenier, J. W., Volten, H., and Muñoz, O.: Scattering matrix of quartz aerosols: Comparison and synthesis of laboratory and Lorenz-Mie results, J. Quant. Spectrosc. Radiat. Transfer, 79-80, 911-920, 2003.

Meng, Z., Yang, P., Kattawar, G., Bi, L., Liou, K., and Laszlo, I.: Single-scattering properties of tri-axial ellipsoidal mineral dust aerosols: A database for application to radiative transfer calcula- 
tions, J. Aerosol Sci., 41, 501-512, 2010.

Mishchenko, M. I.: Light scattering by size-shape distributions of randomly oriented axially symmetric particles of a size comparable to a wavelength, Appl. Opt., 32, 4652-4665, 1993.

Mishchenko, M. I., Travis, L. D., Kahn, R. A., and West, R. A.: Modeling phase functions for dustlike tropospheric aerosols using a shape mixture of randomly oriented polydisperse spheroids, J. Geophys. Res., 102, 16831-16847, 1997.

Mishchenko, M. I., Cairns, B., Kopp, G., Schueler, C., Fafaul, B. A., Hansen, J. E., Hooker, R. J., Itchkawich, T., Maring, H. B., and Travis, L. D.: Accurate Monitoring of Terrestrial Aerosols and Total Solar Irradiance: Introducing the Glory Mission, Bull. Am. Met. Soc., 88, 677-691, 2007.

Muñoz, O., Volten, H., de Haan, J. F., Vassen, W., and Hovenier, J. W.: Experimental determination of scattering matrices of randomly oriented fly ash and clay particles at 442 and $633 \mathrm{~nm}, \mathrm{~J}$. Geophys. Res., 106, 22833-22844, 2001.

Muñoz, O., Volten, H., Hovenier, J., Nousiainen, T., Muinonen, K., Guirado, D., Moreno, F., and Waters, L.: Scattering matrix of large Saharan dust particles: experiments and computations, J. Geophys. Res., 112, D13215, doi:10.1029/2006JD008074, 2007.

Müller, T., Schladitz, A., Massling, A., Kaaden, N., Kandler, K., Wiedensohler, A.: Spectral absorption coefficients and imaginary parts of refractive indices of Saharan dust during SAMUM-1, Tellus B, 61(1), Blackwell Publishing Ltd, 16000889, doi:10.1111/j.1600-0889.2008.00399.x, 79-95, 2009.

Myhre, G. and Stordal, F.: Global sensitivity experiments of the radiative forcing due to mineral aerosols, J. Geophys. Res., 106, 18193-18204, 2001.

Nousiainen, T.: Impact of particle shape on refractive-index dependence of scattering in resonance domain, J. Quant. Spectrosc. Radiat. Transfer, 108, 464-473, doi:10.1016/j.jqsrt.2007.07.008, 2007.

Nousiainen, T.: Optical modeling of mineral dust particles: A review, J. Quant. Spectrosc. Radiat. Transfer, 110, 1261-1279, doi:10.1016/j.jqsrt2009.03.002, 2009.

Nousiainen, T. and Vermeulen, K.: Comparison of measured singlescattering matrix of feldspar particles with $T$-matrix simulations using spheroids, J. Quant. Spectrosc. Radiat. Transfer, 79-80, 1031-1042, 2003.

Nousiainen, T., Kahnert, M., and Veihelmann, B.: Light scattering modeling of small feldspar aerosol particles using polyhedral prisms and spheroids, J. Quant. Spectrosc. Radiat. Transfer, 101, 471-487, doi:10.1016/j.jqsrt.2006.02.038, 2006.
Petzold, A., Rasp, K., Weinzierl, B., Esselborn, M., Hamburger, T., Dörnbrack, A., Kandler, K., Schütz, L., and Knippertz, P., Fiebig, M., and Virkkula, A.: Saharan dust absorption and refractive index from aircraft-based observations during SAMUM 2006, Tellus B, 61(1), Blackwell Publishing Ltd, 1600-0889, doi:10.1111/j.1600-0889.2008.00383.x, 118-130, 2009.

Press, W. H., Teukolsky, S. A., Vetterling, W. T., and Flannery, B. P.: Numerical recipes in FORTRAN, Cambridge University Press, 2 Edn., 1992.

Reid, J. S., Jonsson, H. H., Maring, H. B., Smirnov, A., Savoie, D. L., Cliff, S. S., Reid, E. A., Livingston, J. M., Meier, M. M., Dubovik, O., and Tsay, S.-C.: Comparison of size and morphological measurements of coarse mode dust particles from Africa, J. Geophys. Res., 108, doi:10.1029/2002JD002485, 2003.

Rother, T., Schmidt, K., Wauer, J., Shcherbakov, V., and Gaeyt, J.F.: Light scattering on Chebyshev particles of higher order, Appl. Opt., 45, 6030-6037, 2006.

Schladitz, A., Müller, T., Kaaden, N., Massling, A., Kandler, K., Ebert, M., Weinbruch, S., Deutscher, C., and Wiedensohler, A.: In situ measurements of optical properties at Tinfou (Morocco) during the Saharan Mineral Dust Experiment SAMUM 2006, Tellus B, 61(1), Blackwell Publishing Ltd, 1600-0889, doi:10.1111/j.1600-0889.2008.00397.x, 64-78, 2009.

Schulz, F. M., Stamnes, K., and Stamnes, J. J.: Modeling the radiative transfer properties of media containing particles of moderately and highly elongated shape, Geophys. Res. Lett., 25, 44814484, 1998.

Schulz, F. M., Stamnes, K., and Stamnes, J. J.: Shape-dependence of the optical properties in size-shape distributions of randomly oriented prolate spheroids, including highly elongated shapes, J. Geophys. Res., 104, 9413-9421, 1999.

Sokolik, I. N. and Toon, O. B.: Direct radiative forcing by anthropogenic airborne mineral aerosols, Nature, 381, 681-683, 1996.

van de Hulst, H. C.: Light Scattering by Small Particles, John Wiley \& Sons, 470 pp., 1957.

Veihelmann, B., Nousiainen, T., Kahnert, M., and van der Zande, W. J.: Light scattering by small feldspar particles simulated using the Gaussian random sphere geometry, J. Quant. Spectrosc. Radiat. Transfer, 100, 393-405, 2006.

Volten, H., Muñoz, O., de Haan, J. F., Vassen, W., Hovenier, J. W., Muinonen, K., and Nousiainen, T.: Scattering matrices of mineral aerosol particles at $441.6 \mathrm{~nm}$ and $632.8 \mathrm{~nm}$, J. Geophys. Res., 106, 17375-17401, 2001.

Volten, H., Muñoz, O., Hovenier, J., and Waters, L.: An update of the Amsterdam Light Scattering Database, J. Quant. Spectrosc. Radiat. Transfer, 100, 437-443, 2006. 